\title{
DERIVED SUBDIVISIONS MAKE EVERY PL SPHERE POLYTOPAL
}

\author{
KARIM A. ADIPRASITO AND IVAN IZMESTIEV
}

\begin{abstract}
We give a simple proof that some iterated derived subdivision of every PL sphere is combinatorially equivalent to the boundary of a simplicial polytope, thereby resolving a problem of Billera (personal communication).
\end{abstract}

I. Making any PL sphere polytopal. A subdivision of a simplicial complex $\Delta$ is a simplicial complex $\Delta^{\prime}$ with the same underlying space as $\Delta$, such that for every face $D^{\prime}$ of $\Delta^{\prime}$ there is some face $D$ of $\Delta$ for which $D^{\prime} \subset D$. One also says that $\Delta^{\prime}$ is a refinement of $\Delta$, or writes $\Delta^{\prime} \prec \Delta$. A stellar subdivision of $\Delta$ at a face $\tau$ is defined as

$$
\operatorname{st}(\tau, \Delta):=(\Delta-\tau) \cup\left\{\operatorname{conv}\left\{v_{\tau} \cup \sigma\right\}: \sigma \in \operatorname{St}(\tau, \Delta)-\tau \subset \Delta\right\}
$$

Here $\Delta-\tau$ denotes the deletion of $\tau$ from $\Delta$, i.e. the maximal subcomplex of $\Delta$ that does not contain $\tau$, the point $v_{\tau}$ lies anywhere in the relative interior of $\tau$, and $\operatorname{St}(\tau, \Delta)$ is the star of $\tau$ in $\Delta$, i.e. the minimal subcomplex of $\Delta$ that contains all faces of $\Delta$ containing $\tau$. Clearly, the combinatorial type of the stellar subdivision does not depend on the choice of $v_{\tau}$.

A derived subdivision $\mathrm{sd} \Delta$ is obtained by stellarly subdividing $\Delta$ at all faces in order of decreasing dimension, cf. [Hud69]. A special case is the barycentric subdivision, where the point $v_{\tau}$ is the barycenter of $\tau$.

The derived subdivision can be iterated, by defining $\operatorname{sd}^{m} \Delta:=\operatorname{sd}\left(\operatorname{sd}^{m-1} \Delta\right)$ and $\operatorname{sd}^{0} \Delta:=\Delta$. Our main result in this note is:

Theorem 1. For every PL sphere $\Delta$, there exists a $k \geq 0$ such that $\mathrm{sd}^{k} \Delta$ is polytopal, i.e., it is combinatorially equivalent to the boundary complex of some convex polytope.

Date: March 20, 2014.

2010 Mathematics Subject Classification. 57Q05, 52B70, 52B11.

Key words and phrases. PL sphere; polytopality; derived subdivision.

K. Adiprasito has been supported by an EPDI postdoctoral fellowship and by the Romanian NASR, CNCS-UEFISCDI, project PN-II-ID-PCE-2011-3-0533.

I. Izmestiev has been supported by the European Research Council under the European Union's Seventh Framework Programme (FP7/2007-2013)/ERC Grant agreement no. 247029-SDModels. 
This answers a question asked to the authors on several occasions, in particular by Louis J. Billera (personal communication). The result itself is implicit in the work of Morelli [Mor96, Sec. 6]; however, it was never written up explicitly. We obtain the following immediate corollary, cf. [AB12, Cor. I.3.12]:

Corollary 2. For every closed simplicial $P L$ manifold $M$, there is an $n \geq 0$ such that for every nonempty face $F$ of $\mathrm{sd}^{n} M$, the simplicial $P L$ sphere $\operatorname{Lk}\left(F, \operatorname{sd}^{n} M\right)$ is polytopal.

Proof. Notice that, if $\Delta, \Gamma$ is any pair of PL spheres, then $\operatorname{sd}^{n}(\Delta * \Gamma)$ is a stellar subdivision of $\operatorname{sd}^{n} \Delta * \mathrm{sd}^{n} \Gamma$ (where $*$ denotes the join operation); since stellar subdivisions preserve polytopality, we therefore observe that $\operatorname{sd}^{n}(\Delta * \Gamma)$ is polytopal if $\operatorname{sd}^{n} \Delta$ and $\mathrm{sd}^{n} \Gamma$ are polytopal.

Observe secondly that if $\Delta$ is any simplicial complex, and $F$ is any face of $\operatorname{sd} \Delta$, then there is a face $\widetilde{F} \in \Delta$ and simplices $\sigma_{1}, \cdots, \sigma_{k}$ such that

$$
\operatorname{Lk}(F, \operatorname{sd} \Delta) \cong \operatorname{sd} \partial \sigma_{1} * \cdots * \operatorname{sd} \partial \sigma_{k} * \operatorname{sd} \operatorname{Lk}(\widetilde{F}, \Delta) .
$$

Now, let $n$ be chosen large enough such that for all faces $\widetilde{F}$ of $M$, the complex $\operatorname{sd}^{n} \operatorname{Lk}(\widetilde{F}, M)$ is polytopal. It then follows from the two observations above that for every face $F$ of $\operatorname{sd}^{n} M$, the complex $\operatorname{Lk}\left(F, \operatorname{sd}^{n} M\right)$ is polytopal.

Proof of Theorem 1. A complete pointed fan in $\mathbb{R}^{d+1}$ is a partition of $\mathbb{R}^{d+1}$ into convex polyhedral cones with apices at the origin $\mathbf{0}$ such that the intersection of any two cones is a face of both. A fan is called regular if it consists of the cones over the faces of a convex polytope (with the origin in the interior). A fan $F$ is regular if and only if there exists a PL function $\varphi: \mathbb{R}^{d+1} \rightarrow \mathbb{R}$ whose domains of linearity are exactly the full-dimensional cones of $F$ and that is strictly convex across every codim 1 cone of $F$, cf. [dLRS10]. Thus we have to show that every PL $d$-sphere $\Delta$ becomes combinatorially equivalent to some regular simplicial fan after several derived subdivisions.

We will be repeatedly using the following simple observation:

Lemma 3 (cf. [Zee66, Ch. 1, Lem. 4]). Let $\Delta_{1}$ and $\Delta_{2}$ denote two simplicial complexes with the same support; then there is a derived subdivision $\mathrm{sd}^{k} \Delta_{1}$ that refines $\Delta_{2}$. Moreover, one can choose $k \leq|f|\left(\Delta_{2}\right)$.

Here, $|f|(\cdot)$ denotes the total number of faces of a simplicial complex.

Claim 1: There is an $n$ such that $\operatorname{sd}^{n} \Delta$ is combinatorially equivalent to a simplicial (not necessarily regular) fan in $\mathbb{R}^{d+1}$.

By definition, $\Delta$ is PL homeomorphic to the boundary of the $(d+1)$-simplex $\sigma^{d+1}$. In other words, there are combinatorially equivalent subdivisions $\widetilde{\Delta}$ and $\Sigma$ of $\Delta$ and $\partial \sigma^{d+1}$, respectively. Let now

$$
\vartheta: \widetilde{\Delta} \longrightarrow \Sigma
$$


denote a facewise linear map realizing the combinatorial equivalence, and let $\operatorname{sd}^{n} \Delta$ be chosen fine enough such that $\operatorname{sd}^{n} \Delta \prec \widetilde{\Delta}$. Then $\vartheta\left(\operatorname{sd}^{n} \Delta\right)$ is a subdivision of $\partial \sigma^{d+1}$ combinatorially equivalent to $\operatorname{sd}^{n} \Delta$. The cone with respect to any interior point of $\sigma^{d+1}$ gives the desired simplicial fan.

In the following we identify subdivisions of $\partial \sigma^{d+1}$ with the corresponding fans.

Claim 2: There is a regular subdivision $\Delta^{\prime}$ of $\operatorname{sd}^{n} \Delta$.

Regularity is preserved under stellar, and in particular derived, subdivisions, cf. [dLRS10]. Thus we may take for $\Delta^{\prime}$ any derived subdivision of $\partial \sigma^{d+1}$ that refines $\operatorname{sd}^{n} \Delta$.

Let $\operatorname{sd}^{n+m} \Delta$ be a derived subdivision that refines the regular subdivision $\Delta^{\prime}$ :

$$
\operatorname{sd}^{n+m} \Delta \prec \Delta^{\prime} \prec \operatorname{sd}^{n} \Delta
$$

Claim 3: $\operatorname{sd}^{n+m} \Delta$ is regular.

We have to show that there is a convex PL function with the fan $\operatorname{sd}^{n+m} \Delta$.

First, let us construct a PL function $h: \mathbb{R}^{d+1} \longrightarrow \mathbb{R}$ that is linear on the faces of $\operatorname{sd}^{n+m} \Delta$, and that is strictly convex at every codim 1-face except at the codim 1 skeleton of $\operatorname{sd}^{n} \Delta$.

This is proven by induction: $\operatorname{sd}^{n+m} \Delta$ is a derived, and in particular an iterated stellar subdivision of $\mathrm{sd}^{n} \Delta$; let $\Delta_{1}, \Delta_{2}, \ldots$ denote the intermediate complexes, so that $\Delta_{i+1}$ is obtained from $\Delta_{i}$ using a single stellar subdivision (obtained by introducing a vertex $\nu_{i}$ ).

If $\nu$ is a ray of a simplicial fan $\mathrm{F}$, then let us denote by

$$
[\nu, \mathrm{F}]^{*}(\cdot): \mathbb{R}^{d+1} \longrightarrow \mathbb{R}
$$

the function that is $\langle\cdot, \nu\rangle$ on the ray spanned by $\nu$, that is 0 on all other rays and that is linear on the faces of $\mathrm{F}$. Note that $[\nu, \mathrm{F}]^{*}(\cdot)$ is strictly convex across all codim 1-faces of $F$ that contain $\nu$.

$\mathrm{On} \mathrm{sd}^{n} \Delta=\Delta_{0}$, we just take the zero function.

By induction assumption, let us assume that $\Delta_{i}$ admits a function $h_{i}$ : $\mathbb{R}^{d+1} \longrightarrow \mathbb{R}$ that is linear on faces of $\Delta_{i}$, and strictly convex at every codim 1 face except those in the codim 1 skeleton of $\Delta$. Then, for $\varepsilon_{i}>0$ small enough, the function $\varepsilon_{i}\left[\nu_{i}, \Delta_{i+1}\right]^{*}+h_{i}$ is linear on every face of $\Delta_{i+1}$, and strictly convex at all codim 1 faces newly introduced. Hence, by induction, there is a function $h$ with the desired property.

Now, $\Delta^{\prime}$ is regular, and hence there exists a strictly convex piecewise linear function $h^{\prime}: \mathbb{R}^{d+1} \longrightarrow \mathbb{R}$ whose domains of linearity are the facets of $\Delta^{\prime}$. In particular, $h^{\prime}$ is linear on all faces of $\operatorname{sd}^{n+m} \Delta$. The function $h^{\prime}$ is strictly convex across those faces where the convexity of $h$ can fail. Hence, for an $\varepsilon>0$ small enough, $\varepsilon h+h^{\prime}$ is strictly convex at all codimension one faces of $\mathrm{sd}^{n+m} \Delta$, and linear on all facets of $\mathrm{sd}^{n+m} \Delta$. This finishes the proof. 
II. Algorithmic aspects. Now that we determined that sufficiently many iterations of the derived subdivision make any PL sphere polytopal, it makes sense to ask how many precisely are needed. If $\operatorname{dim} \Delta=2$, then $\Delta$ is combinatorially equivalent to the boundary of a convex polytope by Steinitz Theorem, cf. [Zie95]; the fact that the graph of every triangulation of $S^{2}$ is 3 -connected is an easy exercise. Thus $k=0$ suffices in this case.

For higher dimensions, $k$ can not be bounded that easily, as we shall see now. As usual, $f_{i}(\cdot)$ denotes the number of $i$-dimensional faces of a simplicial complex.

Theorem 4. (a) If $d \geq 3$, then there is no $k$ that would depend only on $d$ such that all $P L d$-spheres become polytopal after $k$ derived subdivisions.

(b) For $d=3$, it number $k=k(\Delta)$ of derived subdivisions needed to make a $P L$ sphere $\Delta$ polytopal can be bounded from above by

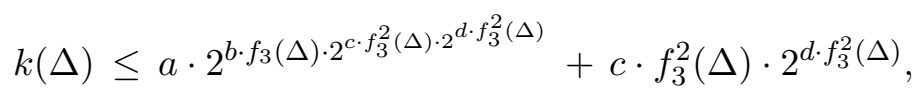

where $a, b, c, d \geq 0$ are constants independent of $\Delta$.

(c) If $d \geq 5$, then the number of derived subdivisions that makes a PL $d$-sphere $\Delta$ polytopal is not (Turing machine) computable from $\Delta$.

In other words, if $\varphi: \mathfrak{K}_{d} \longmapsto \mathbb{N}$ is any computable function (cf. [Dav58]), where $\mathfrak{K}_{d}$ is the collection of $d$-dimensional simplicial complexes, then for some PL $d$-sphere $\Delta$, more than $\Phi(\Delta)$ derived subdivisions are needed to make $\Delta$ polytopal. In particular, the number of subdivisions is not computable from the dimension, the $f$-vector, the flag vector or any other combinatorial invariant of $\Delta$.

Proof. (a) The first statement follows from the work of Bing [Bin64] and Lickorish [Lic91]. Indeed, one can show that for every $d \geq 3$ and every $k \geq 0$, there is a PL $d$-sphere $\Delta$ such that $\operatorname{sd}^{k} \Delta$ is not shellable (cf. [Lic91]). Since the boundary of every convex polytope is shellable [BM71], $\mathrm{sd}^{k} \Delta$ can not be combinatorially equivalent to the boundary of a convex polytope. Compare also [AB12].

(b) For the second assertion, recall that there is an $\ell$ such that $\operatorname{sd}^{\ell} \Delta$ is combinatorially equivalent to a subdivision of (the simplicial fan spanned by) $\partial \sigma^{4}$ by Claim 1 in the proof of Theorem 1. By a result of Mijatović [Mij03], $\ell$ can be bounded in terms of the number of faces of $\Delta$; more explicitly, one can show that $\ell \leq c^{\prime} \cdot f_{3}^{2}(\Delta) \cdot 2^{d^{\prime} \cdot f_{3}^{2}(\Delta)}$, where $c^{\prime}, d^{\prime} \geq 0$ are constants independent of $\Delta$.

Now, there is a iterated derived subdivision $\Delta^{\prime}=\operatorname{sd}^{m} \partial \sigma^{4}$ of $\partial \sigma^{4}$ that is regular and subdivides $\operatorname{sd}^{\ell} \Delta$, and the number of derived subdivisions needed can be bounded from above by

$$
m \leq|f|\left(\operatorname{sd}^{\ell} \Delta\right) \leq(4 !)^{\ell} \cdot 2^{4} \cdot f_{3}(\Delta) .
$$


Finally, the fan $\Delta^{\prime}$ is regular, and there is an $n$ such that $\mathrm{sd}^{\ell+n} \Delta$ subdivides $\Delta^{\prime}$, and

$$
n \leq|f|\left(\Delta^{\prime}\right) \leq(4 !)^{m} \cdot 2^{4} \cdot f_{3}\left(\partial \sigma^{4}\right) .
$$

But $\mathrm{sd}^{\ell+n} \Delta$ is regular by Claim 3 in the proof of Theorem 1 .

(c) For the final claim: suppose there exists a Turing machine computable function $\varphi: \mathfrak{K}_{d} \longmapsto \mathbb{N}$ that, for every PL $d$-sphere $\Delta, d \geq 5$, returns a value $\varphi(\Delta)$ such that $\operatorname{sd}^{\varphi(\Delta)} \Delta$ is polytopal, we would also have Turing machine that decides whether or not a given simplicial $d$-manifold, $d \geq 5$, is a PL sphere: Recall that deciding whether a given simplicial complex is the boundary of a convex polytope is complete within the existential theory of the reals, and therefore Turing machine decidable cf. [Dav58, Mnë88]. Now, if this Turing machine returns, for any $d$-dimensional simplicial complex $\Delta$, that $\operatorname{sd}^{\varphi(\Delta)} \Delta$ is not polytopal, then $\Delta$ is not a PL sphere by assumption; if instead it returns that $\operatorname{sd}^{\varphi(\Delta)} \Delta$ is polytopal, then $\Delta$ is a PL sphere, as desired.

The existence of this Turing machine, however, stands in contradiction to a classical result of S. P. Novikov [VKF74], cf. [Nab95], who proved that it is not decidable whether a given 5-manifold is actually the PL 5-sphere. Therefore, the assumption is wrong, and no such Turing machine bounding $k$ exists.

III. Regular triangulations and geometric bistellar moves. Let $P \subset$ $\mathbb{R}^{d}$ be a convex $d$-polytope. A triangulation $T$ of $P$ (the vertex set of $T$ may be bigger than that of $P$ ) is called regular if there exists a PL function $h: P \rightarrow \mathbb{R}$ linear on all $d$-simplices of $T$ and convex across all of its $(d-1)$ simplices, compare also the notion of a regular fan in the proof of Theorem 1 , and [Zie95] or [dLRS10].

While polytopality is a combinatorial property of a simplicial complex, regularity of a triangulation depends not only on its combinatorics, but also on the position of its vertices.

Theorem 5. For every triangulation $T$ of a convex polytope $P$ there is a $k$ such that some derived subdivision $\mathrm{sd}^{k} T$ is regular.

Proof. The proof is similar to that of Theorem 1. We need a regular triangulation of $P$ to start with: To find one, choose $h_{i} \in \mathbb{R}$ for every vertex $p_{i}$ of $P$ generically and take the lower envelope of the points $\left(p_{i}, h_{i}\right) \in \mathbb{R}^{d+1}$, cf. [dLRS10]. By applying derived subdividisions to $T^{\prime}$, we see that there exists a regular triangulation $T^{\prime}$ of $P$ that refines $T$. Now, there is an $m \geq 0$ such that $\operatorname{sd}^{m} T$ refines $T^{\prime}$. It now follows as in the proof of Theorem 1, Claim 3, that $\mathrm{sd}^{m} T$ is regular.

Corollary 6. Any two triangulations $T_{0}$ and $T_{1}$ of $P$ can be connected by a sequence of geometric Pachner (or bistellar) moves. 
This is essentially the main result of [Mor96] and [Wło97], with the difference that we don't assume $P$ to be a lattice polytope and don't require triangulations to be unimodular. Ewald and Shephard had earlier proven it for regular triangulations [ES74]. On the other hand, Pachner [Pac87] proved that PL homeomorphic manifolds are related by combinatorial Pachner moves.

To deduce Corollary 6 from Theorem 5, take any triangulation $\widetilde{T}$ of $P \times[0,1]$ that restricts to $T_{0}$ and $T_{1}$ on $P \times\{0\}$ and $P \times\{1\}$ respectively, and apply

derived subdivisions to make $\widetilde{T}$ regular. Sweeping out from 0 to 1 produces a sequence of bistellar moves. Details can be found in [IS10, Sec. 2].

Note that geometric bistellar flips (bistellar moves other than stellar subdivisions and moves inverse to them) do not suffice in general to transform one of two triangulations of the same point configuration into the other; see [San05, San06] for a counterexample in dimension 5.

\section{REFERENCES}

[AB12] K. A. Adiprasito and B. Benedetti, Subdivisions, shellability and the Zeeman conjecture, 2012 preprint, available online at arXiv:1202.6606.

[Bin64] R. H. Bing, Some aspects of the topology of 3-manifolds related to the Poincaré conjecture, Lectures on modern mathematics, Vol. II, Wiley, New York, 1964, pp. $93-128$.

[BM71] H. Bruggesser and P. Mani, Shellable decompositions of cells and spheres, Math. Scand. 29 (1971), 197-205 (1972).

[Dav58] M. Davis, Computability and unsolvability, McGraw-Hill Series in Information Processing and Computers, McGraw-Hill Book Co., Inc., New York, 1958.

[dLRS10] J. A. de Loera, J. Rambau, and F. Santos, Triangulations, Algorithms and Computation in Mathematics, vol. 25, Springer-Verlag, Berlin, 2010.

[ES74] G. Ewald and G. C. Shephard, Stellar subdivisions of boundary complexes of convex polytopes., Math. Ann. 210 (1974), 7-16.

[Hud69] J. F. P. Hudson, Piecewise Linear Topology, University of Chicago Lecture Notes, W. A. Benjamin, Inc., New York-Amsterdam, 1969.

[IS10] I. Izmestiev and J.-M. Schlenker, Infinitesimal rigidity of polyhedra with vertices in convex position, Pacific J. Math. 248 (2010), no. 1, 171-190.

[Lic91] W. B. R. Lickorish, Unshellable triangulations of spheres, European J. Combin. 12 (1991), 527-530.

[Mij03] A. Mijatović, Simplifying triangulations of $S^{3}$., Pac. J. Math. 208 (2003), no. 2, 291-324.

[Mnë88] N. E. Mnëv, The universality theorems on the classification problem of configuration varieties and convex polytopes varieties, in "Topology and Geometry-Rohlin Seminar", Lecture Notes in Math., vol. 1346, Springer, Berlin, 1988, pp. 527-543.

[Mor96] R. Morelli, The birational geometry of toric varieties, J. Algebraic Geom. 5 (1996), no. $4,751-782$.

[Nab95] A. Nabutovsky, Einstein structures: Existence versus uniqueness, Geometric \& Functional Analysis GAFA 5 (1995), no. 1, 76-91.

[Pac87] U. Pachner, Konstruktionsmethoden und das kombinatorische Homöomorphieproblem für Triangulationen kompakter semilinearer Mannigfaltigkeiten, Abh. Math. Sem. Univ. Hamburg 57 (1987), 69-86.

[San05] F. Santos, Non-connected toric Hilbert schemes, Math. Ann. 332 (2005), no. 3, 645-665. 
[San06] _ Geometric bistellar flips: the setting, the context and a construction, International Congress of Mathematicians. Vol. III, Eur. Math. Soc., Zürich, 2006, pp. 931-962.

[VKF74] I. A. Volodin, V. E. Kuznecov, and A. T. Fomenko, The problem of the algorithmic discrimination of the standard three-dimensional sphere, Uspehi Mat. Nauk 29 (1974), 71-168, Appendix by S. P. Novikov.

[Wło97] J. Włodarczyk, Decomposition of birational toric maps in blow-ups $\mathcal{G}$ blow-downs, Trans. Amer. Math. Soc. 349 (1997), no. 1, 373-411.

[Zee66] E. C. Zeeman, Seminar on Combinatorial Topology, Institut des Hautes Études Scientifiques, Paris, 1966.

[Zie95] G. M. Ziegler, Lectures on Polytopes, Graduate Texts in Mathematics, vol. 152, Springer, New York, 1995, Revised edition, 1998; seventh updated printing 2007.

Institut des Hautes Études Scientifiques, Bures-sur-Yvette, France

E-mail address: adiprasito@ihes.fr, adiprasito@math.fu-berlin.de

Institut für Mathematik, Freie Universität Berlin, Germany

E-mail address: izmestiev@math.fu-berlin.de 